\title{
Diagnóstico microbiológico en neutropenia febril secundaria a quimioterapia por malignidad hematológica \\ Descripción de una cohorte
}

\author{
Microbiological diagnosis in febrile neutropenia \\ secondary to chemotherapy for hematologic malignancy \\ A cohort description
}

\author{
Karen Andrea García-Rueda, Juliana Londoño-Castillo, Laura Elisa Villegas-Sierra, \\ María Isabel González-Gómez • Medellín (Colombia) \\ Alejandro Correa-García • El Retorno, Guaviare (Colombia)
}

DOI: https://doi.org/10.36104/amc.2020.1386

\section{Resumen}

Objetivo: describir las características demográficas, clínicas, terapéuticas y microbiológicas de los pacientes con malignidad hematológica en quimioterapia que desarrollan neutropenia febril durante el tratamiento, así como la mortalidad al egreso.

Métodos: estudio de cohorte retrospectivo en pacientes con neoplasia hematológica que desarrollaron neutropenia febril evaluados en el Hospital Universitario San Vicente Fundación de Medellín.

Resultados: se obtuvo el registro de 110 episodios de neutropenia febril. Media de edad 45 años. En 55.4\% se obtuvo diagnóstico microbiológico, la bacteriemia fue documentada en $83.8 \%$ de ellos. Los microorganismos más comunes fueron K. pneumoniae (30\%) y E. coli (18\%). La resistencia a antibióticos por presencia de BLEE o carbapenemasas en bacilos gram negativos fue de $33 \%$. La mortalidad ocurrió en $17 \%$ de los pacientes. La leucemia linfoide aguda (LLA) y leucemia mieloide aguda (LMA) fueron los diagnósticos de base más prevalentes, el foco clínico más prevalente fue bacteriemia $(24.5 \%)$.

Conclusión: en nuestra cohorte se encontró diferencias significativas en cuanto al porcentaje de pacientes con bacteriemia, así como en la mortalidad global respecto al resto de cohortes de la región, es necesario desarrollar un nuevo trabajo de investigación que permita aclarar el origen de estas diferencias. (Acta Med Colomb 2020; 45. DOI: https://doi.org/10.36104/amc.2020.1386).

Palabras clave: neutropenia, quimioterapia, microbiología.

\footnotetext{
Abstract

Objective: to describe the demographic, clinical, therapeutic and microbiological characteristics of patients with hematological malignancies undergoing chemotherapy who develop febrile neutropenia during treatment, as well as the mortality at discharge.

Methods: a retrospective cohort study of patients with hematologic neoplasms who developed febrile neutropenia and were seen at the Hospital Universitario San Vicente Fundación in Medellín.

Results: records of 110 episodes of febrile neutropenia were obtained. The mean age was 45 years. In $55.4 \%$, a microbiological diagnosis was obtained; bacteremia was documented in $83.8 \%$ of these. The most common microorganisms were K. pneumoniae (30\%) and E. coli (18\%). Antibiotic resistance due to ESBLs or carbapenemases in gram negative bacilli was $33 \%$. Death occurred in $17 \%$ of patients. Acute lymphocytic leukemia (ALL) and acute myeloid leukemia (AML) were the most prevalent underlying diagnoses; the most prevalent clinical focus was bacteremia $(24.5 \%)$.

Conclusion: in our case series, there are significant differences in the percentage of patients with bacteremia, as well as in global mortality, compared to the rest of the cohorts in the region. A new research study needs to be carried out to clarify the source of these differences. (Acta Med Colomb 2020; 45. DOI: https://doi.org/10.36104/amc.2020.1386).

Key words: neutropenia, chemotherapy, microbiology.
}

Dra. Karen Andrea García-Rueda: Especialista Medicina Interna, Fellowship Cardiología Clínica Universidad de Antioquia; Dra. Juliana Londoño-Castillo: Especialista Medicina Interna, Médico Internista Clínica Las Vegas y SURA EPS y Medicina Prepagada; Dra. Laura Elisa Villegas-Sierra: Médica General Universidad de Antioquia; Dra. María Isabel González-Gómez: Médica y Cirujana Universidad de Antioquia. Medellín (Colombia). Dr. Alejandro Correa-García: Médico General Universidad de Antioquia, Médico de Servicio Social Obligatorio, Hospital de Primer Nivel de El Retorno. Guaviare (Colombia).

Correspondencia. Dra. Karen Andrea GarcíaRueda. Medellín (Colombia).

E-mail: karenandrea0710@gmail.com Recibido: 16/VI/2019 Aceptado: 30/I/2020 


\section{Introducción}

En la actualidad nos encontramos en un momento de notable progreso en la terapia del cáncer y los avances en el tratamiento han incrementado la tasa de supervivencia; dicho avance se debe esencialmente al abordaje terapéutico multimodal, basado en el manejo quirúrgico, la radioterapia, la quimioterapia, la inmunoterapia y el advenimiento de terapias con blancos moleculares dirigida (1). A pesar de lo ya mencionado, hoy en día la quimioterapia con agentes citotóxicos sigue siendo la primera línea de manejo en las neoplasias hematológicas (2). De los diferentes tipos de toxicidad asociada con su uso, los más importantes por su repercusión clínica y por su alta prevalencia son la toxicidad medular y gastrointestinal (3-5). La combinación de estos dos efectos adversos aumenta considerablemente el riesgo de infección en la población bajo tratamiento citotóxico y con ello la mortalidad de este grupo.

En 1966 se describió por primera vez la neutropenia febril, durante los albores de este nuevo síndrome la mortalidad alcanzaba $80 \%$ (6); con el desarrollo de estudios observacionales se logró identificar varias características importantes, a saber, los principales gérmenes causantes eran gram negativos, esencialmente $P$. aeruginosa (7); hacia 1980 con la profilaxis sistemática, además del uso de catéteres venosos centrales, cambió el espectro antimicrobiano hacia un predominio de microorganismos gram positivos. También se estableció que las malignidades hematológicas presentan mayor riesgo de tener esta complicación (10-50\% en pacientes con tumores sólidos versus $80 \%$ en pacientes de hematooncología) (8) y finalmente se determinó que la rápida instauración de antibioticoterapia empírica (al considerar la neutropenia febril como una complicación infecciosa en $100 \%$ de los casos) permitía una reducción de la mortalidad a valores tan bajos como $2.8 \%$ en el presente (9). No obstante, la información concerniente a la epidemiología de las infecciones bacterianas no es estática ni se puede generalizar a todo el globo, por lo que cada cierto tiempo y en cada latitud es necesario conocer la flora propia para realizar el enfoque diagnóstico y terapéutico lo más racional posible.

En Colombia los estudios más recientes datan de hace seis años, con hallazgos importantes, entre los que se destacan la identificación microbiológica en aproximadamente 35-45\% con una prevalencia entre sus aislamientos de infección por microorganismos Gram negativos $(10,11)$. Con dicho aspecto en mente, se planteó la presente cohorte retrospectiva con el objetivo principal de establecer las características microbiológicas de los episodios de neutropenia febril en los pacientes hospitalizados con este diagnóstico en una institución de la ciudad de Medellín.

\section{Material y métodos}

Se realizó un estudio observacional descriptivo de tipo cohorte retrospectiva, de pacientes adultos mayores de 18 años con enfermedad hematológica maligna, en cuyo egreso estuviesen codificados los diagnósticos por código CIE-10: C810-C960, correspondientes a tumores malignos del tejido linfático, de los órganos hematopoyéticos y de tejidos afines y que hubiesen recibido manejo hospitalario en el Hospital San Vicente Fundación de la ciudad de Medellín entre enero de 2013 y diciembre de 2016, de estos, se seleccionaron los episodios (se consideraba como un episodio diferente cada hospitalización, independientemente si el paciente ya había entrado en el estudio por una hospitalización previa) en las que los pacientes hubiesen desarrollado neutropenia febril posquimioterapia. Los datos se recolectaron de los registros electrónicos de historias clínicas. Los investigadores no participaron en el cuidado de los pacientes.

\section{Población}

La población seleccionada debía tener diagnóstico histopatológico de enfermedad hematológica maligna y estar bajo un tratamiento de quimioterapia. Los casos de neutropenia febril se identificaron de acuerdo con los criterios de las definiciones para fiebre y neutropenia de la IDSA (12).

Fiebre: definida como cualquier medición $\geq 38.3^{\circ} \mathrm{C}$ o $\geq 38.0^{\circ} \mathrm{C}$ durante al menos una hora.

Neutropenia: conteo de polimorfonucleares neutrófilos por método de medición directo con número absoluto de neutrófilos $<500$ células/mL.

Se excluyeron pacientes cuyos datos de historia clínica tenían subregistro de variables clínicas, microbiológicas y terapéuticas y también pacientes en quienes se conocía una condición de inmunosupresión concomitante (infección por VIH, tuberculosis pulmonar o extra-pulmonar ya conocida, enfermedades genéticas, diabetes mellitus con mal control metabólico, enfermedad renal terminal definida como tasa de filtración glomerular menor de 15 (TFG <15) o en terapia de reemplazo renal).

En cuanto a las entidades infecciosas se tomaron como definiciones las siguientes:

Bacteriemia: invasión al torrente sanguíneo probada mediante hemocultivos positivos. Divididas en primaria si el único foco clínico y de aislamiento era del torrente sanguíneo y secundaria si la infección inicial se encontraba ubicada en otro órgano (13).

Neumonía: se consideraba tal si había criterio radiológico de neumonía con o sin síntomas (14).

Infección urinaria: diagnosticada por urocultivo positivo aunado a síntomas de infección urinaria alta o baja (15).

Colitis neutropénica: Se definía como tal según el concepto del evaluador clínico o por criterio radiológico cuando estaba disponible $(16,17)$.

Celulitis: se asumía dicho foco si en el examen físico estaba descrito la presencia de un área eritematosa, caliente y dolorosa no explicada por una etiología no infecciosa (18).

Infecciones musculoesqueléticas: Se establecían por criterio radiológico o si se llevaba el paciente a intervención 
quirúrgica en cuya descripción operatoria se registraran signos inflamatorios y/o secreción purulenta proveniente de músculos, tendones, articulaciones o huesos.

\section{Recolección de datos}

Se revisaron historias clínicas contenidas en la base de datos de los servidores del hospital San Vicente Fundación (sistema $\mathrm{SAP} \circledast$ ) por parte de los investigadores del estudio para recolectar datos de variables demográficas, clínicas, microbiológicas y terapéuticas sobre el paciente contenidas en dicho registro clínico, incluyendo: edad, género (hombre o mujer), diagnóstico hematológico (según la clasificación de malignidades hematológicas publicada por la OMS en 2016), esquema de quimioterapia, días desde última sesión de quimioterapia al momento de presentar el episodio de neutropenia febril y condición al egreso (vivo o muerto). En cuanto a las variables microbiológicas se consideraron: microorganismo aislado (en caso de que se hubiese aislado), el sitio de aislamiento (por sistemas) y el método mediante el que se logró el aislamiento (cultivos, molecular, antígenos); en los casos con más de un microorganismo identificado, se consignaron todos y se consideró infección polimicrobiana. Si el aislamiento era un $S$. aureus se registró si había resistencia a la meticilina, igualmente en caso de Enterococcus spp si había resistencia a la vancomicina y en el caso de bacilos gram negativos la presencia de betalactamasa de espectro extendido o de carbapenemasa.
Los datos mencionados, incluida la mortalidad bruta al momento del egreso, obtenidos en el estudio se verificaron por dos investigadores, quienes de manera independiente evaluaron las historias clínicas.

Respecto al control de sesgos por el tipo de estudio, reconocemos que puede haber sesgos de información pues es probable que en los pacientes más graves se hiciese una búsqueda más exhaustiva del agente causal, no hubo pérdidas de seguimiento dado que los desenlaces fueron evaluados hasta el día del egreso.

\section{Análisis estadístico}

Las variables cuantitativas se expresarán como media y rango intercuartil (RIQ). Las variables cualitativas se presentarán como frecuencias absolutas y porcentajes. En cuanto al tratamiento de los datos, se recogieron en el programa Excel@ 2010. El protocolo de investigación fue aprobado por el Comité de Ética del Hospital Universitario San Vicente Fundación.

\section{Resultados}

Se obtuvieron los números de identificación de 1250 episodios potencialmente elegibles y se excluyeron 1140 , principalmente por la ausencia de neutropenia $(n=496)$ o neutropenia febril ( $\mathrm{n}=315)$ (Figura 1).

De los 110 episodios incluidos 50.9\% (n=55) de los pacientes eran hombres, con media de edad 45 años, RIQ 33

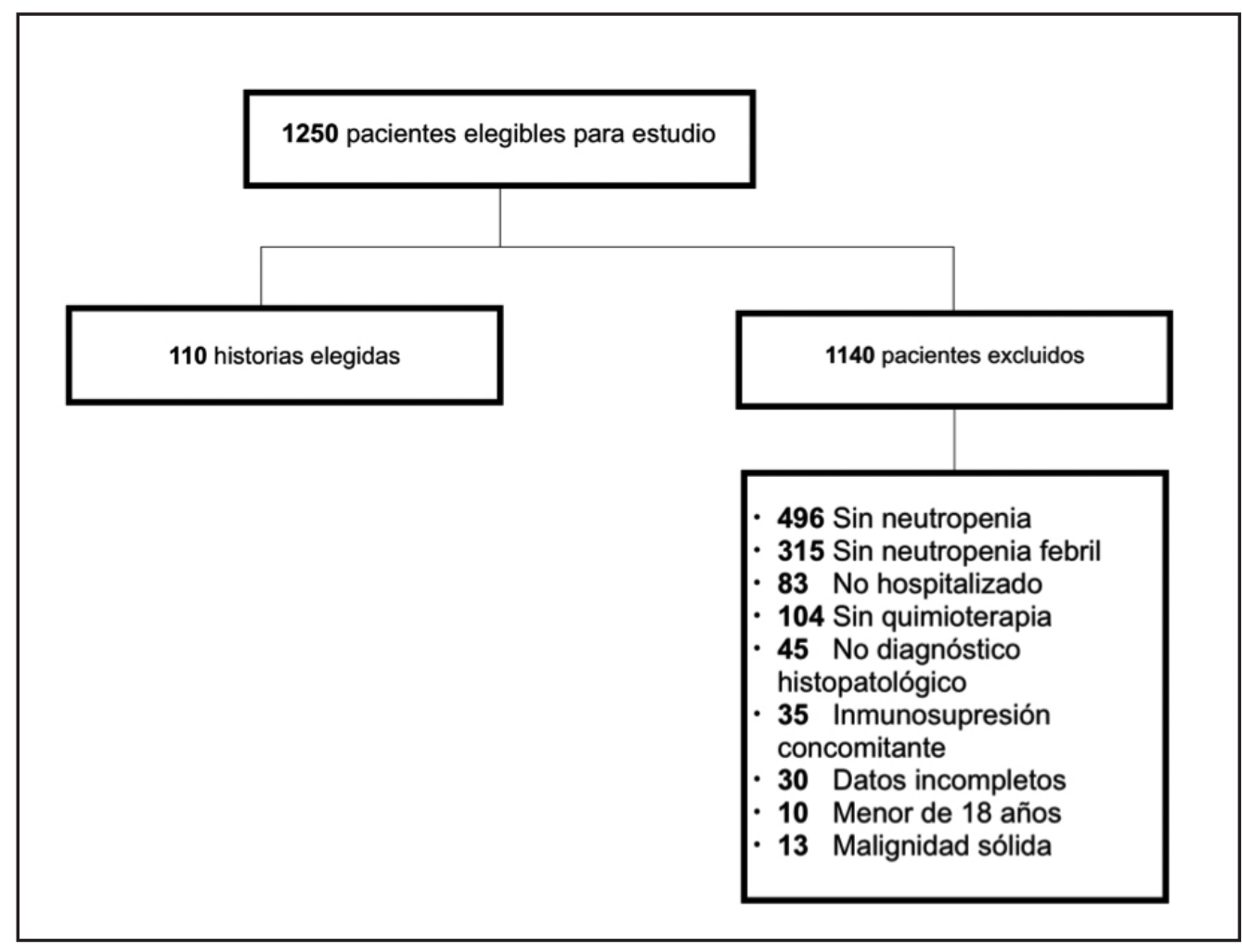

Figura 1. Diagrama de flujo que ilustra la inclusión y exclusión de los participantes en el estudio. 
(Q1 26-Q3 55), los principales diagnósticos hematológicos fueron leucemia linfoide aguda $(\mathrm{n}=29,26.3 \%)$, leucemia mieloide aguda $(\mathrm{n}=29,26.3 \%)$ y linfomas $(\mathrm{n}=27,24.5 \%)$ (Tabla 1). Los principales esquemas de quimioterapia recibidas fueron, en orden de mayor a menor frecuencia, CALBG (daunorrubicina, vincristina, prednisolona, pegasparaginasa, ciclofosfamida) $(n=19,17.27 \%)$, esquema $7+3$ (citarabina, idarrubicina) $(\mathrm{n}=11,10 \%)$ y R-CHOP (prednisona, vincristina, ciclofosfamida, doxorrubicina, rituximab) $(n=10,9.09 \%)$ (Tabla 2). Se estableció un promedio de días desde la última sesión de quimioterapia hasta el diagnóstico de neutropenia de nueve (rango 3-33 días) diferenciado por los principales grupos malignidades: leucemia mieloide aguda: nueve días, leucemia linfoide aguda: nueve días, linfomas: nueve días, neoplasia de células plasmáticas: 10 días. La mortalidad hospitalaria bruta fue $17 \%(\mathrm{n}=19)$. En cuanto a los diagnósticos clínicos: el $25.8 \%(\mathrm{n}=28)$ no tenían ningún foco sospechado, $24.5 \%(\mathrm{n}=27)$ tenían bacteriemia primaria y $19 \%(\mathrm{n}=21)$ neumonía (Tabla 3$)$.

El diagnóstico microbiológico se logró en 55.4\% (n=61) de los episodios, de los cuales el sitio de aislamiento más frecuente fue en sangre con hemocultivos positivos en el $83 \%(\mathrm{n}=51)$, de estas bacteriemias el $53 \%$ fueron primarias $(n=27)$ y el $47 \%(n=24)$ fueron secundarias (Tabla 4); los restantes sitios de aislamiento microbiológico correspondieron

Tabla 1. Diagnóstico hematológico previo al episodio de neutropenia febril en 110 episodios.

\begin{tabular}{|c|c|c|}
\hline Diagnóstico hematológico & $\begin{array}{l}\text { Número de } \\
\text { casos }(\mathbf{n})\end{array}$ & $\begin{array}{l}\text { Porcentaje } \\
(\%)\end{array}$ \\
\hline Leucemia linfoide aguda de células B & 29 & 26.36 \\
\hline Leucemia mieloide aguda & 29 & 26.36 \\
\hline Leucemia promielocitica aguda & 8 & 7.27 \\
\hline Linfoma B difuso de células grandes & 8 & 7.27 \\
\hline Mieloma múltiple & 6 & 5.45 \\
\hline Leucemia linfoide crónica & 4 & 3.64 \\
\hline Linfoma folicular & 4 & 3.64 \\
\hline Linfoma Hodgkin clásico & 4 & 3.64 \\
\hline Leucemia Linfoide aguda de células T & 3 & 2.73 \\
\hline Linfoma Burkitt & 3 & 2.73 \\
\hline Linfoma $\mathrm{T}$ & 3 & 2.73 \\
\hline Leucemia mieloide mrónica & 2 & 1.82 \\
\hline Linfoma B de zona Gris & 2 & 1.82 \\
\hline Linfoma de células del manto & 1 & 0.91 \\
\hline Linfoma de células $\mathrm{T}$ & 1 & 0.91 \\
\hline Linfoma Hodgkincelularidad mixta & 1 & 0.91 \\
\hline $\begin{array}{l}\text { Neoplasia de celulas dendríticas } \\
\text { plasmocitoides } \mathrm{Cd} 4 \mathrm{c}\end{array}$ & 1 & 0.91 \\
\hline Tricoleucemia & 1 & 0.91 \\
\hline
\end{tabular}

Tabla 2. Esquema de quimioterapia administrada previamente al episodio de neutropenia febril.

\begin{tabular}{|c|c|c|}
\hline Esquema de Quimioterapia* & $\begin{array}{l}\text { Número de } \\
\text { casos (n) }\end{array}$ & $\begin{array}{c}\text { Porcentaje } \\
(\%)\end{array}$ \\
\hline CALGB & 19 & 17.27 \\
\hline $7+3$ & 11 & 10 \\
\hline R-CHOP & 10 & 9.09 \\
\hline HYPERCVAD & 8 & 7.27 \\
\hline GRALL 2003 & 7 & 6.36 \\
\hline ABVD & 2 & 1.82 \\
\hline HIDAC & 2 & 1.82 \\
\hline R-EPOCH & 2 & 1.82 \\
\hline FLAG.IDA & 1 & 0.91 \\
\hline ICE & 1 & 0.91 \\
\hline RBENDAMUSTINE & 1 & 0.91 \\
\hline FRALLE & 0 & 0 \\
\hline PETHEMA & 0 & 0 \\
\hline CODOX M & 0 & 0 \\
\hline OTROS & 46 & 41.82 \\
\hline \multicolumn{3}{|c|}{$\begin{array}{l}\text { *CALGB (daunorubicina, vincristina, prednisolona, peg-asparaginasa, cyclofosfamida); } \\
\text { 7+3 (citarabina, idarrubicina); R-CHOP (prednisona,vincristina, ciclofosfamida, doxo- } \\
\text { rrubicina, rituximab); HYPERCVAD (ciclo A: ciclofosfamida, vincristina, doxorubicina, } \\
\text { dexametasona ciclo B: metotrexate + citarabina); GRALL } 2003 \text { (pre-fase prednisolona, } \\
\text { metotrexate; vincristina, prednisolona, daunorrubicina, asparaginasa, ciclofosfamida); } \\
\text { ABVD (doxorubicina, bleomicina, vinblastina, dacarbazina); HIDAC (altas dosis de } \\
\text { citarabina); R-EPOCH (rituximab, etopósido, prednisona, vincristina, ciclofosfamida y } \\
\text { doxorrubicina); FLAG. IDA (fludarabina, citarabina, GC-SCF, Idarrubicina); ICE (Ifos- } \\
\text { famida, Carboplatino, Etopósido); R-BENDAMUSTINE (Bendamustine-Rituximab); } \\
\text { FRALLE (prednisona, vincristina, daunorrubicina, asparaginasa); PETHEMA (idarrubi- } \\
\text { cina, acido todo-transretinoico); CODOX-M (ciclofosfamida, vincristina, doxorrubicina, } \\
\text { dosis altas de metotrexate). }\end{array}$} \\
\hline
\end{tabular}

Tabla 3. Diagnóstico clínico al momento del diagnóstico de neutropenia febril.

\begin{tabular}{|l|c|c|}
\hline \multicolumn{1}{|c|}{ Diagnóstico clínico } & $\begin{array}{c}\text { Número de } \\
\text { casos (n) }\end{array}$ & $\begin{array}{c}\text { Porcentaje } \\
(\%)\end{array}$ \\
\hline Bacteriemia primaria & 27 & 24.5 \\
\hline Neumonía & 21 & 19 \\
\hline Colitis neutropénica & 17 & 15.4 \\
\hline Celulitis & 9 & 8.1 \\
\hline Infección urinaria & 6 & 5.4 \\
\hline Sistema musculoesquelético & 2 & 1.8 \\
\hline Sin diagnóstico clínico ni aislamiento & 28 & 25.8 \\
\hline
\end{tabular}

Tabla 4. Foco clínico en bacteriemias secundarias.

\begin{tabular}{|l|c|c|}
\hline \multicolumn{1}{|c|}{ Diagnóstico clínico } & $\begin{array}{c}\text { Número de } \\
\text { casos }(\mathbf{n})\end{array}$ & $\begin{array}{c}\text { Porcentaje } \\
(\boldsymbol{\%})\end{array}$ \\
\hline Colitis neutropénica & 10 & 41 \\
\hline Neumonía & 6 & 25 \\
\hline Celulitis & 4 & 16 \\
\hline Infección urinaria & 3 & 12.5 \\
\hline Osteomuscular & 1 & 4.1 \\
\hline
\end{tabular}


a sistema genitourinario en $11 \%(\mathrm{n}=7)$, cavidad abdominal y tracto gastrointestinal en $8.1 \%(n=5)$ (dos abscesos intraabdominales con muestra obtenida mediante aspiración percutánea y tres por aislamiento en materia fecal), piel y tejidos blandos y tracto respiratorio, cada uno con un paciente (en ambos casos infecciones fúngicas)

Los principales microorganismos fueron $K$. pneumoniae en $30 \%$ de los aislamientos $(n=20), 19$ cursaron con bacteriemia y uno con infección urinaria; E. coli en $18.4 \%$ de los aislamientos $(n=12)$, con ocho bacteriemias, dos aislamientos en tracto gastrointestinal (aspiración de absceso intraabdominal) y dos infecciones urinarias. S. aureus con bacteriemia en ocho de los aislamientos (12\%).

Respecto a las infecciones fúngicas, el aislamiento se documentó en cuatro episodios: En tres de estos se diagnosticó candidiasis invasora, dos de ellos con evidencia de fungemia por Cándida tropicalis, el tercero con evidencia de candidiasis hepatoesplénica con aislamiento microbiológico en cultivo de micosis profunda en piel obtenida a partir de biopsia. Sólo hubo un diagnóstico definitivo de aspergillosis invasora mediante aislamiento por cultivo de biopsia de tabique nasal (considerado como aislamiento a partir de tracto respiratorio) (Figura 2). Se documentaron siete episodios de aspergilosis pulmonar invasiva probable detectados por antigenemia para aspergillus e imagen tomográfica compatible.

El porcentaje de infección polimicrobiana fue de $12 \%$ $(n=11)$; el 100\% se aisló en sangre con al menos un bacilo gram negativo, predominando K. pneumoniae en el $81.8 \%$ de los casos de $(n=9)$. En $54 \%$ de estos episodios $(n=6)$ había diagnóstico de aspergilosis pulmonar invasiva probable (todos estos con K. pneumoniae como agente concomitante, una de estas con patrón BLEE, otra con producción de carbapenemasa y el resto silvestres).

Relativo al patrón de resistencia (Tabla 5), los bacilos gram negativos $23,8 \%$ tuvieron presencia de BLEE $(n=10)$ de los cuales el $60 \%(n=6)$ recibieron antibióticos en los 30 días previos (33.3\% recibió quinolonas y $66.6 \%$ betalactámicos). Además $9.5 \%(n=4)$ de los aislamientos con bacilos gram negativos presentaron producción de carbapenemasas; de éstos el $100 \%(n=4)$ estuvieron expuestos a tratamiento antibiótico en los 30 días previos (100\% carbapenémicos, $75 \%$ piperacilina/tazobactam y $25 \%$ quinolonas).

En los casos en los que se aisló S. aureus $25 \%$ de ellos fueron resistentes a meticilina, ninguno de estos pacientes había tenido exposición previa a antibióticos (Tabla 6).

Ninguno de los pacientes previamente mencionados estaba en profilaxis con trimetoprim sulfametoxasol, fluconazol o ciprofloxacina.

\section{Discusión}

La presente cohorte de pacientes con malignidad hematológica bajo quimioterapia que desarrollaron neutropenia febril con requerimiento de hospitalización contó con un número similar de pacientes a otras ya reportadas en Colombia y América Latina. La edad media de presentación fue de 45 años, la relación hombre: mujer fue 1:1, en consonancia con estudios realizados en Colombia (11), Chile

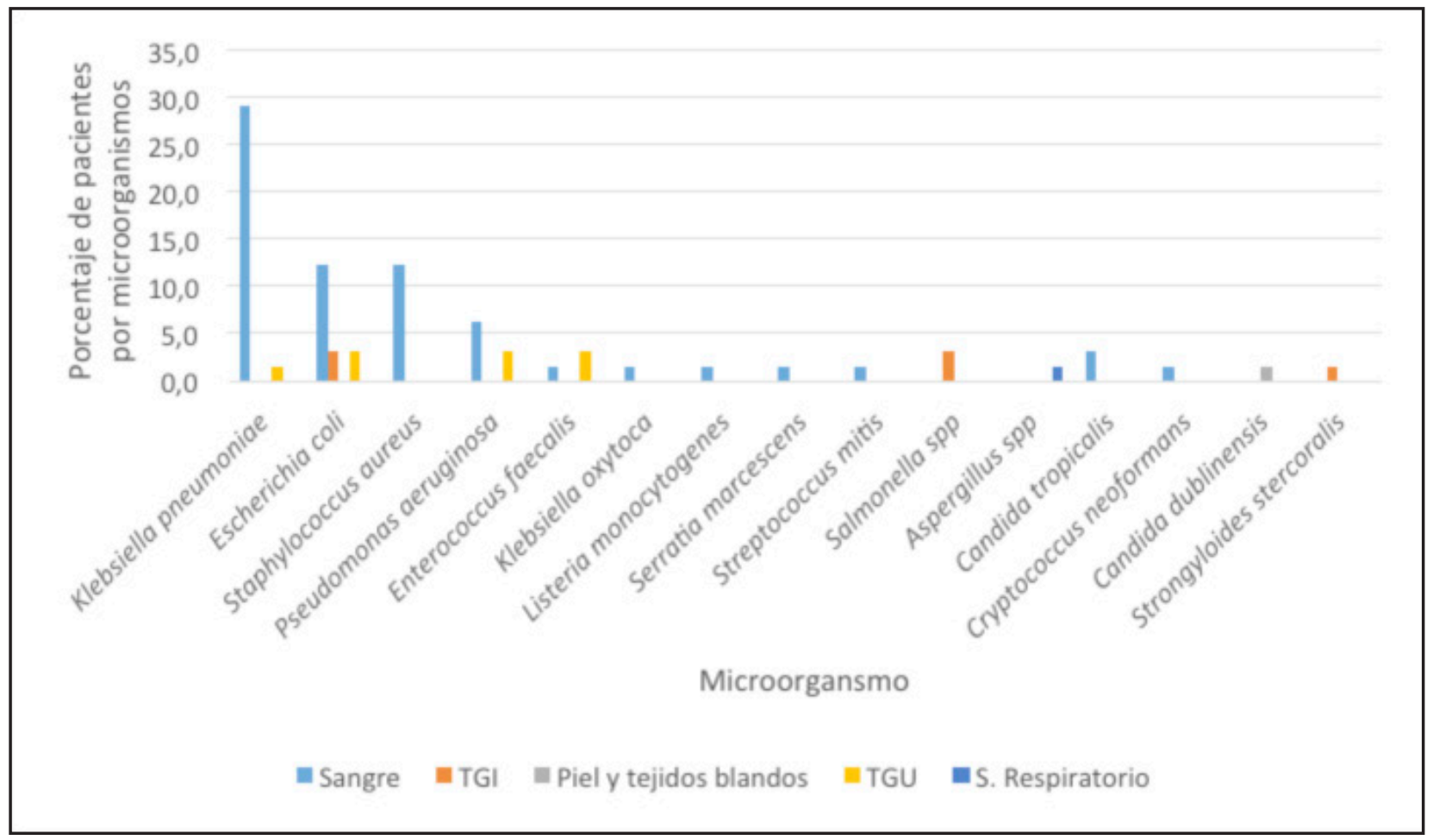

Figura 2. Identificación microbiológica y sitios de aislamiento en pacientes con neutropenia febril. (TGI: tracto gastrointestinal; TGU: tracto genitourinario 
Tabla 5. Perfiles de resistencia antimicrobiana en microorganismos gram negativos.

\begin{tabular}{|l|c|c|}
\hline Microorganismo (n) & $\begin{array}{c}\text { Betalactamasa de } \\
\text { espectro extendido (n) }\end{array}$ & $\begin{array}{c}\text { Carbapene- } \\
\text { masa (n) }\end{array}$ \\
\hline E. coli $(12)$ & 2 & 0 \\
\hline K.oxytoca $(1)$ & 0 & 0 \\
\hline K. pneumoniae (20) & 7 & 4 \\
\hline P. aeruginosa (6) & 1 & 0 \\
\hline Salmonella spp (2) & 0 & 0 \\
\hline S. marcescens $(1)$ & 0 & 0 \\
\hline
\end{tabular}

Tabla 6. Perfiles de resistencia antimicrobiana en microorganismos gram positivos.

\begin{tabular}{|l|c|c|}
\hline Microorganismo (n) & $\begin{array}{c}\text { Resistencia a } \\
\text { meticilina }\end{array}$ & $\begin{array}{c}\text { Resistencia a } \\
\text { vancomicina }\end{array}$ \\
\hline S. aureus $(8)$ & 2 & 0 \\
\hline
\end{tabular}

(19) y Cuba (20). Con respeto al diagnóstico oncológico de base, la mayoría de cohortes regionales fueron basadas en pacientes con malignidades tanto sólidas como hematológicas; en búsqueda de una población más homogénea se decidió realizar el estudio solo basado en población con malignidades hematológicas, al igual que el estudio realizado en el Hospital Pablo Tobón Uribe en MedellínColombia (11). Al compararlos, se encontraron diferencias en las condiciones oncológicas de base de estas cohortes, así el principal diagnóstico oncológico en dicho estudio fue leucemia mieloide aguda, en el nuestro hubo una prevalencia por igual de leucemia linfoide aguda y leucemia mieloide aguda (cada una con $26.3 \%$ ) en tercer y cuarto lugar leucemia promielocítica y linfoma difuso de células B ( $7.2 \%$ en cada caso), la quimioterapia más relacionada en el mismo estudio fue HIDAC, seguida de HYPERCVAD, en nuestro CALGB (17.2\%) seguida de 7+3 (10\%); aunque esto representa diferencias en cuanto al riesgo de neutropenia febril, dado que en ambos estudios se tuvieron en consideración sólo los pacientes que ya la presentaban no consideramos que haya diferencias en cuanto a los resultados secundarias a este aspecto. En cuanto a los diagnósticos clínicos en estos pacientes, en la cohorte que presentamos la mayoría de pacientes no tenían un foco clínico sospechado al igual que en la cohorte del Hospital Pablo Tobón, si bien las proporciones son diferentes ( 25.4 vs $63.5 \%$ ). Como dato relevante, la mortalidad obtenida en nuestro estudio fue de $17 \%$, lo cual dista de $7 \%$ de la mayoría de cohortes de esta región $(11,19,20)$, lo cual indica la necesidad de explorar otras condiciones para aclarar el origen de dicha diferencia.

Respecto a los hallazgos microbiológicos se encontró que en $55.4 \%$ de los episodios de neutropenia febril que tuvieron lugar en el periodo comprendido entre enero de 2013 y diciembre de 2016, se logró aislar un agente microbiológico como causa de dicho cuadro clínico, a nivel regional los diagnósticos microbiológicos oscilan entre el 41 y $66 \%$ de los casos (21); los dos focos clínicos con más aportes al aislamiento microbiológico fueron la infección urinaria (11\%) y bacteriemias $(83 \%)$ lo cual dista de forma importante de la mayoría de estudios publicados tanto a nivel local, regional y mundial, donde la bacteriemia solo aporta el 20-30\% de los hallazgos (12); en cuanto a los lugares donde se presume inicio la infección que da origen a la bacteriemia son tracto gastro-intestinal, tracto respiratorio, tracto genitourinario y piel (10), lo cual es coincidente con lo que encontramos.

Respecto a los agentes microbiológicos, resulta relevante señalar que hasta 1980 la mayoría de aislamientos en estos pacientes eran gram-negativos (siendo especialmente relevante $P$. aeuruginosa), desde esa fecha el perfil microbiológico viró hacia los micro-organismos gram positivos (especialmente los coagulasa negativos) lo cual probablemente fuese explicado por el uso de catéteres venosos centrales y el uso de antimicrobianos con espectro dirigido hacia gram negativos. Desde principios del actual siglo, nuevamente hay un cambio microbiológico lo cual se ve representado en las otras cohortes nacionales, con predominio de bacilos gram negativos $(10,11)$, así los agentes con mayor número de aislamientos en estas cohortes fueron E.coli y K. pneumoniae; en nuestro estudio la distribución de gérmenes entre bacterias gram negativas, bacterias gram positivas y hongos fue de $68.8,21.3$ y $8.1 \%$ respectivamente. El microorganismo con mayor número de aislamientos fue $K$. pneumoniae . Es llamativa la presencia de coinfección con bacilos gram negativos y hongos, específicamente de Aspergillus con $K$. pneumoniae, aunque es una muestra pequeña este hallazgo no es despreciable.

En lo relativo a resistencia antibiótica, en los estudios de la región, la prevalencia de betalactamasa de espectro extendido oscila entre el 7 y $26 \%(10,11)$, en nuestro hospital fue de 23\%; en estos mismos estudios la resistencia de $S$. aureus a meticilina fue de $25 \%$ al igual que lo encontrado por nosotros.

Reconocemos como limitaciones del presente estudio su carácter retrospectivo con el sesgo de información que esto suele acarrear, no obstante, dado que los hallazgos que son marcadamente diferentes con respecto a estudios previos, específicamente el número de pacientes con bacteriemia documentada, son obtenidos de registros objetivos (esto es resultados de laboratorio) y que el procesamiento de hemocultivos es una práctica recomendada por las guías de manejo de neutropenia febril (12), consideramos que dicho sesgo pudo no haber influido en los resultados. En cuanto a las variables recolectadas, se reconoce que no tener el resultado de la duración de la neutropenia y así poder discriminar la población en neutropenia prolongada o no constituye una limitación significativa para interpretar el número de aislamientos no piógenos; sin embargo, los otros trabajos publicados que si presentan diferenciación tienen resultados similares en proporción, lo que orienta a que probablemente estemos en el mismo rango de pacientes con neutropenia prolongada (22). Adicionalmente, dado el alto porcentaje 
de pacientes con bacteriemia documentada, hubiese sido deseable la diferenciación entre bacteriemia primaria versus asociada al catéter lo cual exigía haber conservado el mismo sistema operativo para el procesamiento de muestras; así mismo dada la alta mortalidad hubiese sido conveniente haber consignado el riesgo de muerte al momento del diagnóstico de neutropenia febril (por ejemplo mediante la escala MASCC); sin embargo, dado que por ser pacientes hematológicos que requirieron manejo en hospitalización son considerados de alto riesgo, al plantear el proyecto no tuvimos en cuenta dicha consideración.

En cuanto a las fortalezas, destacamos el tamaño de la muestra que fue similar a la mayoría de cohortes publicadas a nivel regional, el origen de los datos al pertenecer a un hospital de cuarto nivel donde el estudio y manejo de la neutropenia febril se puede realizar de forma exhaustiva dada la disponibilidad de recursos, se cuenta con fuentes de información homogénea, y las características de la población que son así mismo homogéneas (por tener todos malignidad hematológica) y con el mayor número de factores de confusión excluidos (por ejemplo el diagnóstico de VIH, la presencia de diabetes mellitus no controlada) permite considerar que su susceptibilidad a infecciones y los microorganismos aislados se encuentran todos en íntima relación con el proceso oncológico y la quimioterapia administrada.

Consideramos que se contribuye al conocimiento de los aspectos microbiológicos de los pacientes con neutropenia febril, en esencia encontramos características muy similares a lo documentado en el resto de publicaciones; sin embargo, es relevante destacar la elevada prevalencia de bacteriemia y la alta mortalidad, que consideramos amerita una nueva investigación que permita establecer las diferencias que llevan a dicho descubrimiento, probablemente mediante un estudio comparativo entre las características demográficas y clínicas no exploradas hasta ahora (por ejemplo la presencia de escalofríos, entre otros). Dadas las características metodológicas del estudio y las limitaciones ya mencionadas no es posible dar recomendaciones con implicación clínica directa; no obstante, si permite motivar a nuevos campos de investigación que permitan un mejor abordaje de estos pacientes (22).

\section{Agradecimientos}

Agradecemos a la Dra. Diana Carolina Moncada, especialista en Medicina Interna y en Enfermedades Infecciosas, a la Dra. Isabel Cristina Ramírez, especialista en Medicina Interna y en Enfermedades Infecciosas y al Dr. Sergio Andrés Bedoya especialista en Medicina Interna y en Oncología Clínica, por sus invaluables aportes a la elaboración de este manuscrito.

\section{Financiación}

Declaramos que no se recibió financiación de ninguna fuente.

\section{Referencias}

1. Ke X, Shen L. Frontiers in Laboratory Medicine Molecular targeted therapy of cancer : The progress and future prospect. Front Lab Med. 2017;1(2):69-75.

2. Hassanpour SH, Dehghani M. Review of cancer from perspective of molecular. J Cancer Res Pract. 2017;4(4):127-9.

3. Liu S, Kurzrock R. Toxicity of targeted therapy: Implications for response and impact of genetic polymorphisms. Cancer Treat Rev. 2014;40(7):883-91.

4. Liu S, Kurzrock R. Understanding Toxicities of Targeted Agents_Implications for Anti-tumor Activity and Management. Semin Oncol. 2015;42(6):863-75.

5. Dy G,Adjei A. Understanding, Recognizing, and Managing Toxicities of Targeted Anticancer Therapies. CA Cancer J Clin. 2013;63(4):249-79.

6. Swati M, Gita N. Microbial Etiology of Febrile Neutropenia. Indian J Hematol Blood Transfus. 2010;26(June):49-55.

7. Samonis, G., Vardakas, K.Z., Maraki, S. et al. A prospective study of characteristics and outcomes of bacteremia in patients with solid organ or hematologic malignancies. Support Care Cancer 2013;21(April): 2521-2526

8. Bodey GP, Buckley M, Sathe YS, Ph D. Leukocytes and Infection in Patients with Acute Leukemia. Ann Intern Med. 1966;64(2):328-42.

9. Flowers CR, Seidenfeld J, Bow EJ, Karten C, Gleason C, Hawley DK, et al. Antimicrobial Prophylaxis and Outpatient Management of Fever and Neutropenia in Adults Treated for Malignancy : American Society of Clinical Oncology Clinical Practice Guideline. J Clin Oncol. 2019;31(6):794-810.

10. Cortés JA, Cuervo S, Gómez CA, Bermúdez D, Martínez T, Arroyo P, et al. Neutropenia febril en el trópico: una descripción de los hallazgos clínicos y microbiológicos y el impacto de la terapia inapropiada que utilizan en un centro de referencia oncológica en Colombia. Biomédica. 2012;33(1):70-77.

11. Madrid C, Díaz L, Combariza J, Gálvez K, Olaya V, Ramírez I, et al. Epidemiología de la neutropenia febril en pacientes adultos con neoplasia hematológica, en un período de 26 meses en el Hospital Pablo Tobón Uribe, Colombia. Rev Chil infectología. 2013;30(2):195-201.

12. Freifeld AG, Bow EJ, Sepkowitz KA, Boeckh MJ, Ito JI, Mullen CA, et al. Clinical practice guideline for the use of antimicrobial agents in neutropenic patients with cancer: 2010 Update by the Infectious Diseases Society of America. Clin Infect Dis. 2011;52(4):e56-93.

13. Ruiz-Giardin JM, Noguerado Asensio A. Bacteriemias. An Med Interna 2005;22(3): 105-107.

14. Evans, Scott E.; Ost, David E. Pneumonia in the neutropenic cancer patient. Curr Opin Pulm Med. 2015;21(3): 260-271.

15. Tancheva, S., Micheva, I., Marinova, I., Bojchev, B., Marinov, M., Nenov, K.S., et al. Infections in urinary tract of patients with haematological malignancies undergoing antineoplastic therapy. JofIMAB 2009;15(3):95-97

16. Rolston KVI, Bodey GP, Safdar A. Polymicrobial Infection in Patients with Cancer: An Underappreciated and Underreported Entity. Clin Infec Dis. 2007;45(2):228-33.

17. NHI. Oral Complications of Cancer and Cancer Therapy : From Cancer Treatment to Survivorship Oral Complications of Cancer and Cancer Therapy From Cancer Treatment to Survivorship [Internet]. 2019. Available from: https://www.cancer. gov/about-cancer/treatment/side-effects/mouth-throat/oral-complications-pdq

18. Pulia MS, Calderone MR, Meister JR, Santistevan J, May L. Update on management of skin and soft tissue infections in the emergency department. Curr Infect Dis Rep. 2014;16(9):418-427.

19. Rabagliati R, Bertín P, Cerón I, Rojas H, Domínguez I, Vera Á, et al. Epidemiología de neutropenia febril en pacientes adultos con leucemia aguda y linfoma. Estudio de cohorte en hospitales público y privado de Santiago, Chile. Rev Chil Infectol. 2012;31(6):721-8.

20. Ricardo D, Ávila M, González HS, Reyes S. Febrile neutropenia after cytostatic therapy observed in the Institute of Oncology and Radiobiology. Rev Cub Farm. 2016;50(1):44-52.

21. Rabagliati B Ricardo, Fuentes L Gino, Orellana U Eric, Oporto C Jorge, Domínguez M Isabel, Benítez G Rosana et al. Etiología de episodios de neutropenia febril en pacientes adultos con cáncer hematológico y de órganos sólidos en el Hospital Clínico Universidad Católica, Santiago-Chile. Rev Chil Infectol. 2009;26(2):106-13.

22. Schimpff SC, Young VMAE, Ph D, Greene WH, Vermeulen GD, Moody MR, et al. Origin of Infection in Acute Nonlymphocytic Leukemia Significance of Hospital Acquisition of Potential Pathogens. Ann Intern Med. 1972;77(5):707-14. 